\title{
On the Multiscale Nature of Human Prostate Cancer
}

\section{Fabio Grizzi*}

Humanitas Research Hospital, Via Manzoni 56 - 20089 Rozzano, Milan, Italy

Among men, cancers of the prostate, lung and bronchus, and colorectum will account for about $50 \%$ of all newly diagnosed cancers. Prostate cancer alone will account for $27 \%$ of incident cases in men [1]. Prostate-specific antigen (PSA) is one of the most widely used tumor markers and will continue to be a first line biomarker for prostate cancer management, but it has severe limitations mainly due to its low specificity and low negative predictive value [2]. Today, a new generation of biomarkers is emerging, consisting of serum-, urine-, and tissue-based assays that may supplement PSA testing, or replace it over time [3]. Diagnostic biomarkers should be sensitive and specific enough to show whether disease is present; however, the levels of most biomarkers overlap between tumor and normal tissues [4]. Consequently, it remains difficult to identify an ideal biomarker with enough discriminatory power for an accurate diagnosis of prostate cancer [4]. Histological evaluation remains the gold standard. The improvements in biopsies have resulted in an increase in early-stage tumor detection, although this procedure is highly invasive, offers a low level of accuracy (i.e. only 30\% detection rate at the first biopsy) and is prone to various complications [5]. It has been reported that the major challenge for any molecular test is its ability to reliably detect mutations in samples consisting of mixtures of tumor cells and their normal counterparts [6]. Morphologically, prostate cancers look alike, even though they may be vastly different at the molecular and behavioral level. Tumors histologically may be composed of hundreds of "molecular disease" subsets observed for genomic aberrations [7]. The conception of anatomical entities as a hierarchy of infinitely graduated forms and the increase in the number of observed anatomical subentities and structural variables has generated a growing complexity, thus highlighting new proprieties of organized biological matter [8]. Every anatomical tissue is capable of expressing a particular function in a particular context and its different "configurations" emerge from structures organized in overlapping hierarchical levels [8]. It must be emphasized that observed morphological patterns might often be conceptualized as "macro-scale manifestations" of "microscale processes". Observed "patterns" are generated or influenced by multiple processes and controls, which operate at multiple "spatial" and "temporal" scales, both larger and smaller than the scale of observation [9]. It is known that prostate cancer is increasingly as being a highly heterogeneous disease. Likely to other solid tumors, this heterogeneity is manifested at both genetic and phenotypic level, and primarily determines the "self-progression" of neoplastic disease and its response to therapy $[10,11]$. High-throughput sequencing of tumor samples has shown that most tumors exhibit extensive intra-tumor heterogeneity, with multiple subpopulations of tumor cells containing different somatic mutations [12]. Tumors progress through a sequential series of genetic alterations but the order of these alterations can vary among tumors and even among different areas of the same tumor. The dynamics of prostate cancer is discontinuous in "space" and "time". It advances through qualitatively different "states", i.e. the configuration patterns that are specified by a great number of dynamic variables. The parameter "time"” depends on a large number of variables that are non-linearly interconnected in a multitude of ways, thus making it extremely difficult to predict the exact time interval between two successive states [13]. It has been showed that the decisive step in prostate carcinogenesis is the result of an irreversible qualitative change in one or more of the genetic characteristics of cancer cells, and that tumors have somatic mutations and epigenetic changes $[4,14]$.
Although this modification governs the transformation of normal human cells into malignant cancer cells, it may or may not lead to visible changes in their "cytological" or "histological" structures [15]. In men with an initial negative prostate biopsy and with a persistent suspicious of prostate cancer microscopically normal but genetically altered cells may acquire mutations or epigenetic alterations that can initiate the development of a tumor of the same or a different histological type. Analysis of the initiation and progression of prostate cancer cells from their normal counterparts and the intra-tumor heterogeneity raises two questions: $a$ ) what are the proprieties shared by cancer and natural cells? And $b$ ) to what extent are these proprieties shared? This can be explained using the concept of "emergence", which defines a biological tissue as a "complex system" consisting of different cell types that are interconnected at many organizational levels, have various degree of complexity, and are governed by specific laws that only operate at a particular level [16]. Emergence is a seminal concept that denotes the principle that the "emergent" global proprieties defining higher order system, i.e. tissue or "wholes" cannot generally be reduced to the properties of the lower order subsystem or "parts", i.e. cells. We shall here use the word "emergence" to mean the appearance of unexpected structures and / or the occurrence of surprising behaviors in tissue consisting of sub-microscopic non-identical parts: i.e. structures and behaviors that are not intuitive or simply predictable. This "multiscale causality" not only recognizes multiple processes and controls acting at multiple scales but, unlike a strict reductionist approach, may also recognize the fact that relevant "first principles" may reside at scales other than that the smallest micro-scales. In other words, the observed phenomenon at each scale has structural and behavioral proprieties that do not exist at lower or higher organizational levels [17]. In addition, although each of the spatial scales may have multiple temporal scales, biological process that take place at a lower scale generally happen much faster than those at a higher scale [16]. It is now clear that "prostate cancer" admits many descriptions (ways of looking at the system), each of which is only partially true. Each way of looking at a "cancer system" requires its own description, its own mode of analysis and its own breakdown into different parts. Histology or genetic tests alone may not adequately segregate tumors for supporting clinical decisions. Therefore a multi-level approach makes it possible the recognition of neoplastic cells disguised using a single technique. Application of these concepts promises to be useful for analyzing and modeling prostate cancer development and progression. Looking prostate cancer behavior across multiple biological scales in space and time promise not only to refine hypothesis, focus experiments and more accurate predictions, but also improve our abilities to define more efficient prostate cancer therapeutic strategies.

*Corresponding author: Fabio Grizzi, Humanitas Clinical and Research Center Via Manzoni 56 - 20089 Rozzano, Milan, Italy, Tel: +39 02 82245262; Fax: +3902 82244590; E-mail: fabio.grizzi@humanitasresearch.it

Received July 10, 2014; Accepted July 11, 2014; Published July 20, 2014

Citation: Grizzi F (2014) On the Multiscale Nature of Human Prostate Cancer. Pharmaceut Reg Affairs 3: e135. doi:10.4172/2167-7689.1000e135

Copyright: (c) 2014 Grizzi F. This is an open-access article distributed under the terms of the Creative Commons Attribution License, which permits unrestricted use, distribution, and reproduction in any medium, provided the original author and source are credited. 


\section{References}

1. Siegel R, Ma J, Zou Z, Jemal A (2014) Cancer statistics, 2014. CA Cancer J Clin 64: 9-29.

2. Stephan C, Ralla B, Jung K (2014) Prostate-specific antigen and other serum and urine markers in prostate cancer. Biochim Biophys Acta 1846: 99-112.

3. Crawford ED, Ventii K, Shore ND (2014) New biomarkers in prostate cancer. Oncology (Williston Park) 28: 135-142.

4. Paziewska A, Dabrowska M, Goryca K, Antoniewicz A, Dobruch J, et al. (2014) DNA methylation status is more reliable than gene expression at detecting cancer in prostate biopsy. Br J Cancer .

5. Raaijmakers R, Kirkels WJ, Roobol MJ, Wildhagen MF, Schrder FH (2002) Complication rates and risk factors of 5802 transrectal ultrasound-guided sextant biopsies of the prostate within a population-based screening program. Urology 60: 826-830.

6. Smits AJ, Kummer JA, de Bruin PC, Bol M, van den Tweel JG, et al. (2014) The estimation of tumor cell percentage for molecular testing by pathologists is not accurate. Mod Pathol 27: 168-174.

7. Munoz J, Swanton C, Kurzrock R (2013) Molecular profiling and the reclassification of cancer: divide and conquer. Am Soc Clin Oncol Educ Book .

8. Grizzi F, Chiriva-Internati M (2005) The complexity of anatomical systems. Theor Biol Med Model 2: 26.
9. Masoudi-Nejad A, Bidkhori G, Hosseini Ashtiani S, Najafi A, Bozorgmehr JH, et al. (2014) Cancer systems biology and modeling: Microscopic scale and multiscale approaches. Semin Cancer Biol .

10. Marusyk A, Almendro V, Polyak K (2012) Intra-tumour heterogeneity: a looking glass for cancer? Nat Rev Cancer 12: 323-334.

11. Almendro V, Marusyk A, Polyak K (2013) Cellular heterogeneity and molecular evolution in cancer. Annu Rev Pathol 8: 277-302.

12. Hajirasouliha I, Mahmoody A, Raphael BJ (2014) A combinatorial approach for analyzing intra-tumor heterogeneity from high-throughput sequencing data. Bioinformatics 30: i78-78i86.

13. Taverna G, Seveso M, Giusti G, Hurle R, Graziotti P, et al. (2014) Senescent remodeling of the innate and adaptive immune system in the elderly men with prostate cancer. Curr Gerontol Geriatr Res 2014: 478126.

14. Bhavsar T, McCue P, Birbe R (2013) Molecular diagnosis of prostate cancer: are we up to age? Semin Oncol 40: 259-275.

15. Berman DM, Epstein Jl (2014) When is prostate cancer really cancer? Urol Clin North Am 41: 339-346.

16. Deisboeck TS, Wang Z, Macklin P, Cristini V (2011) Multiscale cancer modeling Annu Rev Biomed Eng 13: 127-155.

17. Grizzi F, Chiriva-Internati M (2006) Cancer: looking for simplicity and finding complexity. Cancer Cell Int 6: 4 